Pacific Journal of Mathematic 


\title{
AN ELEMENTARY PROOF OF THE UNIQUENESS OF THE FIXED POINT INDEX
}

\author{
ROBERT F. BROWN
}

In 1953, Barrett O'Neill stated axioms for a "fixed point index" and obtained existence and uniqueness theorems for the index on finite polyhedra. The proof of uniqueness consisted of showing that any function which satisfied the axioms must agree with the index he had already defined. This paper presents a proof of the uniqueness of the fixed point index on finite polyhedra which depends only on the axioms and therefore is "elementary" in the sense that it is independent of the existence of an index. The proof is "elementary" also in that all the techniques used are taken from geometric topology or calculus so that, in particular, no algebraic topology is required. An elementary proof of the uniqueness of the fixed point index on compact metric absolute neighborhood retracts is an immediate consequence of the material in this paper.

1. The axioms. Let $\mathscr{C}$ be a collection of topological spaces. Denote by $\mathscr{C}^{\prime}$ the collection of all triples $(X, f, U)$ where $X$ is in $\mathscr{C}, f: X \rightarrow X$ is a map, and $U$ is an open subset of $X$ such that there are no fixed points of $f$ on the boundary of $U$.

A fixed point index on $\mathscr{C}$ is a function $i: \mathscr{C}^{\prime} \rightarrow Z$ (the integers) such that

I. (Localization). If $(X, f, U) \in \mathscr{C}^{\prime}$ and $g: X \rightarrow X$ is a map such that $g(x)=f(x)$ for all $x$ in the closure of $U$, then $i(X, f, U)=i(X, g, U)$.

II. (Homotopy). Given a map $H: X \times I \rightarrow X$, define $h_{t}: X \rightarrow X$, for $t \in I=[0,1]$, by $f_{t}(x)=H(x, t)$. If $\left(X, h_{t}, U\right) \in \mathscr{C}^{\prime}$ for all $t \in I$, then $i\left(X, h_{0}, U\right)=i\left(X, h_{1}, U\right)$.

III. (Additivity). If $(X, f, U) \in \mathscr{C}^{\prime}$ and $U_{1}, \cdots, U_{s}$ is a set of mutually disjoint open subsets of $U$ such that $f(x) \neq x$ for all $x \in\left[U-\bigcup_{j=1}^{s} U_{j}\right]$, then $i(X, f, U)=\sum_{j=1}^{s} i\left(X, f, U_{j}\right)$.

IV. (Weak Normalization). If $(X, f, U) \in \mathscr{C}^{\prime}$ where $f$ is the constant map such that $f(X)=x_{0} \in U$, then $i(X, f, U)=1$.

V. (Commutativity). If $X$ and $X^{\prime}$ are in $\mathscr{C}$ and $f: X \rightarrow X^{\prime}$, $g: X^{\prime} \rightarrow X$ are maps such that $(X, g f, U) \in \mathscr{C}^{\prime}$ then $i(X, g f, U)=$ $i\left(X^{\prime}, f g, g^{-1}(U)\right)$.

The axiom list above is a modified version of the one used by Browder in [1]. It differs somewhat from O'Neill's original list [5].

2. Proof of uniqueness. Given a space $X$ and a function $f: X \rightarrow R^{n}$ (euclidean $n$-dimensional space), we will always denote by 
the corresponding capital letter $F: X \rightarrow R^{n}$ the function such that $F(x)=x-f(x)$ for all $x \in X$. Let $O$ be the origin in $R^{n}$.

Lemma 1. If $U$ is an open subset of $R^{n}$ containing $O$ and $f$ : $U \rightarrow R^{n}$ is a linear function whose only fixed point is $\boldsymbol{O}$, then $\partial F(\boldsymbol{O}) / \partial\left(x_{1}, \cdots, x_{n}\right)$ (the Jacobian of $F$ at $\left.\boldsymbol{O}\right)$ is not zero.

Proof. Suppose that $\partial F(\boldsymbol{O}) / \partial\left(x_{1}, \cdots, x_{n}\right)=0$. Since $f$ is linear on $U$ and $f(\boldsymbol{O})=\boldsymbol{O}$, there exists a linear transformation $g: R^{n} \rightarrow R^{n}$ such that $g(x)=f(x)$ for all $x \in U$. We observe that $G: R^{n} \rightarrow R^{n}$ is also a linear transformation so, letting $d G$ denote the differential of $G$, we have $d G(x)=G$ for all $x \in R^{n}$. Since $G$ is identical with $F$ in a neighborhood of $\boldsymbol{O}$, the determinant of $G$ is

$$
\operatorname{det}(G)=\frac{\partial G(\boldsymbol{O})}{\partial\left(x_{1}, \cdots, x_{n}\right)}=\frac{\partial F(\boldsymbol{O})}{\partial\left(x_{1}, \cdots, x_{n}\right)}=0
$$

and $G$ is a singular linear transformation. Therefore, there exists a vector subspace $S$ of $R^{n}$, of dimension at least one, such that $G(S)=O$. Thus, for $x \in S \cap U$, we have

$$
0=G(x)=x-g(x)=x-f(x)
$$

which means that the points of $S \cap U$ are fixed points of $f$, in contradiction to the hypothesis that $O$ is the only fixed point.

For $r \neq 0$ a real number, define $\sigma(r)=1$ if $r>0$ and $\sigma(r)=-1$ if $r<0$.

If $f: X \rightarrow X$ is a map, $x$ is a fixed point of $f$, and there exists an open set $V$ in $X$ containing $x$ such that the closure of $V$ contains no other fixed point of $f$, then define the index of $f$ at $x$ by $i(X, f, x)=$ $i(X, f, V)$. The additivity axiom implies that the definition is independent of the choice of $V$.

Theorem 2. Let $\boldsymbol{O} \in U \subset X \subset R^{n}$ where $U$ is open in $R^{n}$ and $X$ is a finite polyhedron. If $f: X \rightarrow X$ is of class $C^{1}$ on $U, f(\boldsymbol{O})=\boldsymbol{O}$, and $\partial F(\boldsymbol{O}) / \partial\left(x_{1}, \cdots, x_{n}\right) \neq 0$, then $i(X, f, \boldsymbol{O})=\sigma\left(\partial F(\boldsymbol{O}) / \partial\left(x_{1}, \cdots, x_{n}\right)\right)$ for any index $i$ on finite polyhedra.

The proof of this theorem, using only the axioms, is given in the next section.

Let $K$ be a finite simplicial complex and let $|K|$ denote its geometric realization. A pair $T=(K, \tau)$, where $\tau=|K| \rightarrow X$ is a homeomorphism, is a triangulation of the polyhedron $X$. A simplicial map 
$g:\left(X, T_{1}\right) \rightarrow\left(X, T_{2}\right)$ is a map induced by a simplicial function from $K_{1}$ to $K_{2}$. A maximal simplex of $T=(K, \tau)$ is a subset $\tau|s|$ of $X$ where $|s|$ is an open simplex of $|K|$ which is not a face of any other simplex.

THEOREM 3 (Hopf [3]). Let $X$ be a finite polyhedron and $f: X \rightarrow X$ a map. Given $\varepsilon>0$ there exist triangulations $T_{1}$ and $T_{2}$ of $X$ and a simplicial approximation $g:\left(X, T_{1}\right) \rightarrow\left(X, T_{2}\right)$ to $f$, whose distance from $f$ is less than $\varepsilon$, such that all fixed points of $g$ are in maximal simplices of $T_{1}$.

We are now able to prove the uniqueness of the fixed point index on finite polyhedra.

Let $\mathscr{C}$ be the collection of all finite polyhedra and suppose that $i: \mathscr{C}^{\prime} \rightarrow Z$ is a fixed point index. Given $(X, f, U) \in \mathscr{C}^{\prime}$, we will show how to compute $i(X, f, U)$ directly from the axioms and that will imply that the index, if it exists, is unique.

Since the boundary of $U$ is compact and contains no fixed point of $f$, there exists $\varepsilon>0$ such that, for all $x$ in the boundary of $U$, the distance from $x$ to $f^{\prime}(x)$ is greater than $\varepsilon$. Applying Theorem 3, we obtain a simplicial approximation $g:\left(X, T_{1}\right) \rightarrow\left(X, T_{2}\right)$ to $f$. Recall that consequently there is a homotopy $h_{t}: X \rightarrow X, t \in I$, along line segments in $X$ such that $h_{0}=f$ and $h_{1}=g$. Since the distance from $f$ to $g$ is less than $\varepsilon$, the distance from $f$ to each $h_{t}$ is less than $\varepsilon$ so $\left(X, h_{t}, U\right) \in \mathscr{C}^{\prime}$ and, by the homotopy axiom, $i(X, f, U)=i(X, g, U)$.

If a single maximal simplex of $T_{1}$ contained two fixed points of $g$, then since $g$ is linear on each closed simplex of $T_{1}, g$ would be the identity map on the line segment (in the closure of the maximal simplex) which is determined by the two points. But the segment would intersect the boundary of the simplex, which is a union of nonmaximal simplices. Thus there would be a nonmaximal simplex of $T_{1}$ containing a fixed point of $g$, contrary to Theorem 3 . Each maximal simplex of $T_{1}$, therefore, contains at most one fixed point of $g$ and $g$ has only a finite number of fixed points. Let $x_{1}, \cdots, x_{r}$ be the fixed points of $g$ in $U$, then, by the additivity axiom, $i(X, g, U)=$ $\sum_{j=1}^{r} i\left(X, g, x_{j}\right)$.

Therefore, we need only consider the following situation. We have an open subset $\tau_{1}|s|$ of $X$ homeomorphic to $R^{n}$ for some $n \geqq 1$ (because $\tau_{1}|s|$ is a maximal simplex of $T_{1}$ ) and a map $g: X \rightarrow X$ which is linear on $\tau_{1}|s|$ and which has a single fixed point $x$ in $\tau_{1}|s|$. Let $\bar{V}$ be a closed $n$-cell in $\tau_{1}|s|$ such that $x \in V$ and $g(\bar{V}) \subset \tau_{1}|s|$. Choose a closed $n$-cell $Y$ in $\tau_{1}|s|$ containing $\bar{V} \cup g(\bar{V})$. There are retractions $\rho_{\alpha}: Y \rightarrow \bar{V}$ and $\rho_{\beta}: X \rightarrow Y$. Let $\alpha=g \rho_{\alpha}: Y \rightarrow Y$ and $\beta=\alpha \rho_{\beta}: X \rightarrow X$. The localization axiom tells us that 


$$
i(X, g, x)=i(X, g, V)=i(X, \beta, V) \text {. }
$$

Furthermore, for $j: Y \rightarrow X$ the inclusion map, the commutativity axiom implies

$$
\begin{aligned}
i(X, \beta, V) & =i\left(X, j \alpha \rho_{\beta}, V\right) \\
& =i\left(Y, \alpha \rho_{\beta} j, j^{-1}(V)\right) \\
& =i(Y, \alpha, V)
\end{aligned}
$$

Observe that $\alpha(x)=g(x)$ for all $x \in V$, so $\alpha$ is linear on $\Lambda$.

There is a linear homeomorphism $h$ taking $\tau_{1}|s|$ onto an open subset of $R^{n}$ such that $h(x)=\boldsymbol{O}$. Let $a=h \alpha h^{-1}: h(Y) \rightarrow h(Y)$ then, by the commutativity axiom again,

$$
i(Y, \alpha, V)=i(h(Y), a, h(V))=i(h(Y), a, \boldsymbol{O}) .
$$

Since $a$ is linear on $h(V),\left(\partial A(O) /\left(\partial\left(x_{1}, \cdots, x_{n}\right)\right) \neq 0\right.$ by Lemma 1 and so, by Theorem 2 ,

$$
i(X, g, x)=i(h(Y), a, \boldsymbol{O})=\sigma\left(\frac{\partial A(\boldsymbol{O})}{\partial\left(x_{1}, \cdots, x_{n}\right)}\right) .
$$

Therefore, we will have completed our elementary proof of the uniqueness of the fixed point index on finite polyhedra once we prove Theorem 2 directly from the axioms in the next section.

We have not only proved uniqueness but also, in the process, constructed a candidate for a fixed point index. Given $(X, f, U) \in \mathscr{C}^{\prime}$, we have the map $g$ as above with fixed points $x_{1}, \cdots, x_{r}$ in $U$ and we could define

$$
i(X, f, U)=\sum_{j=1}^{r} \sigma\left(\frac{\partial A_{j}(\boldsymbol{O})}{\partial\left(x_{1}, \cdots, x_{n(j)}\right)}\right)
$$

where $A_{j}$ is defined like the map $A$ above and $n(j)$ is the dimension of the maximal simplex of $T_{1}$ containing $x_{j}$. However, we would still be required to verify that this function $i$ really satisfies the axioms. Since an elegant proof of the existence of a fixed point index on finite polyhedra is given in the first two sections of [2], such a verification would appear to be of little interest.

We note that our uniqueness proof together with the proof of Lemma 0 of [1] constitutes an elementary proof of the uniqueness of the fixed point index on the collection of all compact metric absolute neighborhood retracts.

3. Proof of Theorem 2. The result we are setting out to prove is not new (see [4, p. 214]). What is new, however, is a proof which depends only on the axioms for a fixed point index. The first 
step is a proof of Theorem 2 in the case $n=1$.

Lemma 4. If $0 \in(a, b) \subset R^{1}, f:[a, b] \rightarrow[a, b]$ is differentiable at $0, f(0)=0$ and $d F(0) / d x>0$, then there exists $\varepsilon>0$ and a homotopy $h_{t}:[a, b] \rightarrow[a, b], t \in I$, such that $h_{0}=f, h_{1} \operatorname{maps}[a, b]$ to 0 and $h_{t}(\varepsilon) \neq \varepsilon$, $h_{t}(-\varepsilon) \neq-\varepsilon$ for all $t \in I$.

Proof. Since $d F(0) / d x>0$ then $d f(0) / d x<1$ so there exists $\varepsilon>0$ such that $0<|x| \leqq \varepsilon$ implies $\left(f^{\prime}(x)-f(0)\right) / x<1$. Therefore, $f(x)<x$ if $0<x \leqq \varepsilon$ and $f^{\prime}(x)>x$ if $-\varepsilon \leqq x<0$. Define $h_{t}(x)=(1-t) f^{\prime}(x)$ for $x \in[a, b]$.

Lemma 5. Let $0 \in(a, b) \subset R^{1}, f:[a, b] \rightarrow[a, b]$ be a map differentiable at $0, f(0)=0$ and $d F(0) / d x \neq 0$. If $i: \mathscr{C}^{\prime} \rightarrow Z$ is any index on finite polyhedra, then $i([a, b], f, 0)=\sigma(d F(0) / d x)$.

Proof. If $d F(0) / d x>0$, then using Lemma 4 and the homotopy axiom,

$$
\begin{aligned}
i([a, b], f, 0) & =i([a, b], f,(-\varepsilon, \varepsilon)) \\
& =i\left([a, b], h_{1},(-\varepsilon, \varepsilon)\right)
\end{aligned}
$$

where $h_{1}$ is the constant map at 0 so $i\left([a, b], h_{1},(-\varepsilon, \varepsilon)\right)=1$ by the weak normalization axiom. If $d F(0) / d x<0$ then there exists $\varepsilon>0$ such that $f(-\varepsilon)<-\varepsilon$ and $f(\varepsilon)>\varepsilon$. Define a map $g:[a, b] \rightarrow[a, b]$ as follows. If $x \in[-\varepsilon, \varepsilon]$, let $g(x)=f(x)$. Choose $c \in(\varepsilon, f(\varepsilon))$, define $g(f(\varepsilon))=c$ and extend $g$ linearly over $[\varepsilon, f(\varepsilon)]$. Finally, extend $g$ over $[a, b]$. By the additivity axiom

$$
i([a, b], g,(-\varepsilon, f(\varepsilon)))=i([a, b], g,(-\varepsilon, \varepsilon))+i([a, b], g,(\varepsilon, f(\varepsilon)) .
$$

Since $g(\varepsilon)>\varepsilon$ and $g(f(\varepsilon))<f(\varepsilon)$, an argument like the one in Lemma 4 and the first part of this proof shows that $i([a, b], g,(\varepsilon, f(\varepsilon)))=1$. Next observe that $g(-\varepsilon)<-\varepsilon$ and $g(f(\varepsilon))<f(\varepsilon)$ so, choosing $e \in(g(-\varepsilon),-\varepsilon)$, the homotopy $h_{t}(x)=(1-t) g(x)+t e$ for $x \in[a, b]$ has the properties $h_{0}=g, h_{1}$ is the constant map at $e$, and $h_{t}(-\varepsilon) \neq-\varepsilon$, $h_{t}(f(\varepsilon)) \neq f(\varepsilon)$ for all $t \in I$. Therefore, by the homotopy axiom,

$$
i([a, b], g,(-\varepsilon, f(\varepsilon)))=i\left([a, b], h_{1},(-\varepsilon, f(\varepsilon))\right) .
$$

However, $h_{1}$ has no fixed points on $[-\varepsilon, f(\varepsilon)]$ so, by the additivity axiom, $i\left([a, b], h_{1},(-\varepsilon, f(\varepsilon))\right)=0$. Consequently, $i([a, b], g,(-\varepsilon, \varepsilon))=-1$ and, by the localization axiom, $i([a, b], f,(-\varepsilon, \varepsilon))=-1$.

Lemma 6. If, for $j=1,2,0 \in\left(a_{j}, b_{j}\right) \subset R^{1}, f_{j}:\left[a_{j}, b_{j}\right] \rightarrow\left[a_{j}, b_{j}\right]$ is differentiable at $0, f_{j}(0)=0$ and $d F_{j}(0) / d x<0$, then there exists $\varepsilon>0$ 
and a homotopy $h_{t}:\left[a_{1}, b_{1}\right] \times\left[a_{2}, b_{2}\right] \rightarrow\left[a_{1}, b_{1}\right] \times\left[a_{2}, b_{2}\right]$ such that $h_{0}(x, y)=$ $\left(f_{1} \times f_{2}\right)(x, y)=\left(f_{1}(x), f_{2}(y)\right), h_{1}$ is the constant map at $\boldsymbol{O}$, and $h_{t}(p) \neq p$ for all $t \in I$ and all $p$ on the boundary of $V=[-\varepsilon, \varepsilon] \times[-\varepsilon, \varepsilon]$.

Proof. The hypothesis $d F_{j}(0) / d x<0$ implies that there exists $\varepsilon>0$ such that $f_{j}(x)>x$ for $0<x \leqq \varepsilon$ and $f_{j}(x)<x$ when $-\varepsilon \leqq x<0$. Let $B_{\varepsilon}$ denote the closed ball in $R^{2}$ of radius $\varepsilon$ centered at $O$ and let $S_{\varepsilon}$ be the boundary of $B_{\varepsilon}$. Define $r: V \rightarrow B_{\varepsilon}$ to be the retraction such that if $p \notin B_{\varepsilon}$ then $r(p)$ is the point where the ray from $O$ through $p$ intersects $S_{\varepsilon}$. Define $s: B_{\varepsilon} \rightarrow V$ to be radial projection. If $p \in B_{\varepsilon}$ then $p=\alpha e^{i \theta}$ for some $\alpha \in[0, \varepsilon]$ and $\theta \in[0,2 \pi)$. For a real number $t$, define $\rho_{t}: B_{\varepsilon} \rightarrow B_{\varepsilon}$ to be the rotation $\rho_{t}\left(\alpha e^{i \theta}\right)=\alpha e^{i(\theta+t \pi)}$. Finally, define $h_{t}: V \rightarrow\left[a_{1}, b_{1}\right] \times\left[a_{2}, b_{2}\right]$ as follows

$$
h_{t}(p)= \begin{cases}\left(f_{1} \times f_{2}\right) s \rho_{t} r(p) & 0 \leqq t \leqq 1 / 2 \\ 2(1-t) h_{1 / 2}(p) & 1 / 2 \leqq t \leqq 1\end{cases}
$$

and extend $h_{t}$ to $h_{t}:\left[a_{1}, b_{1}\right] \times\left[a_{2}, b_{2}\right] \rightarrow\left[a_{1}, b_{1}\right] \times\left[a_{2}, b_{2}\right]$ so that $h_{0}=f_{1} \times f_{2}$ and $h_{1}$ is constant.

Lemma 7. Let $X, Y$, and $X \times Y$ be spaces in a collection $\mathscr{C}$ and let $i: \mathscr{C}^{\prime} \rightarrow Z$ be an index. If $f: X \rightarrow X$ is a map with an isolated fixed point at $x_{0}$ and $k: Y \rightarrow Y$ is the constant map such that $k(Y)=y_{0}$, then $i\left(X \times Y, f \times k,\left(x_{0}, y_{0}\right)\right)=i\left(X, f, x_{0}\right)$.

Proof. Define $\pi: X \times Y \rightarrow X$ by $\pi(x, y)=x$ and $j: X \rightarrow X \times Y$ by $j(x)=\left(x, y_{0}\right)$. Let $U$ be an open subset of $X$ containing $x_{0}$ whose closure contains no other fixed point of $f$. By the commutativity axiom,

$$
\begin{aligned}
i\left(X \times Y, f \times k,\left(x_{0}, y_{0}\right)\right) & =i(X \times Y, f \times k, U \times Y) \\
& =i(X \times Y, j \pi(f \times k), U \times Y) \\
& =i\left(X, \pi(f \times k) j, j^{-1}(U \times Y)\right) \\
& =i(X, f, U)=i\left(X, f, x_{0}\right) .
\end{aligned}
$$

Next we prove Theorem 2 for a special kind of map.

Lemma 8. For $j=1, \cdots, n$, let $0 \in\left(a_{j}, b_{j}\right) \subset R^{1}, f_{j}:\left[a_{j}, b_{j}\right] \rightarrow\left[a_{j}, b_{j}\right]$ be maps differentiable at $0, f_{j}(0)=0$, and $d F_{j}(0) / d x \neq 0$. Let $W=$ $\Pi_{j=1}^{n}\left[\alpha_{j}, b_{j}\right] \subset R^{n}$ and $f=f_{1} \times \cdots \times f_{n}$, then

$$
i(W, f, \boldsymbol{O})=\sigma\left(\frac{\partial F(\boldsymbol{O})}{\partial\left(x_{1}, \cdots, x_{n}\right)}\right)
$$

where $i: \mathscr{C}^{\prime} \rightarrow Z$ is any index on finite polyhedra. 
Proof. Certainly,

$$
\frac{\partial F(\boldsymbol{O})}{\partial\left(x_{1}, \cdots, x_{n}\right)}=\frac{d F_{1}(0)}{d x} \cdots \frac{d F_{n}(0)}{d x} .
$$

By the commutativity axiom, $i(W, f, \boldsymbol{O})$ is independent of the ordering of the $f_{j}$ 's, so assume $d F_{j}(0) / d x$ is negative for $j=1, \cdots, m$ and positive for $j=m+1, \cdots, n$ (where it may be that $m=0$ or $m=n$ ). Then we see that $\sigma\left(\partial F(\boldsymbol{O}) / \partial\left(x_{1}, \cdots, x_{n}\right)\right)=(-1)^{m}$. Write $m=2 r+\delta$ where $\delta=0$ or $\delta=1$. Applying Lemma $4 n-m$ times and Lemma $6 r$ times, we obtain $\varepsilon>0$ and a homotopy $h_{t}: W \rightarrow W, t \in I$, such that $h_{0}=f, h_{1}$ is the constant map taking $W$ to $\boldsymbol{O} \in R^{n}$ if $\delta=0, h_{1}=$ $f_{1} \times k$ where $k$ maps $\Pi_{j=2}^{n}\left[a_{j}, b_{j}\right]$ to $\boldsymbol{O} \in R^{n-1}$ if $\delta=1$, and $h_{t}(p) \neq p$ $p$ on the boundary of $[-\varepsilon, \varepsilon] \times \cdots \times[-\varepsilon, \varepsilon] \subset R^{n}$. Thus, if $m$ is even,

$$
i(W, f, \boldsymbol{O})=i\left(W, h_{1}, \boldsymbol{O}\right)=1
$$

by the weak normalization axiom and, if $m$ is odd,

$$
\begin{aligned}
i(W, f, \boldsymbol{O}) & =i\left(W, f_{1} \times k, \boldsymbol{O}\right) \\
& =i\left(\left[a_{1}, b_{1}\right], f_{1}, 0\right) \\
& =-1
\end{aligned}
$$

by Lemmas 5 and 7 . In either case, $i(W, f, \boldsymbol{O})=(-1)^{m}$ which completes the proof.

Lemma 9. Suppose $0 \in\left(a_{j}, b_{j}\right) \subset R^{1}$ for $j=1, \cdots, n$ and let $W=$ $\Pi_{j=1}^{n}\left[a_{j}, b_{j}\right]$. Let $f: W \rightarrow W$ be a map such that $f(\boldsymbol{O})=\boldsymbol{O}, f$ is of class $C^{1}$ on a neighborhood of $\boldsymbol{O}$ and $\partial F(\boldsymbol{O}) / \partial\left(x_{1}, \cdots, x_{n}\right) \neq 0$. There exists a homotopy $h_{t}: W \rightarrow W$ and an open subset $V$ of $W$ containing $O$ such that $h_{0}=f, h_{1}=g_{1} \times \cdots \times g_{n}$ where $g_{j}:\left[a_{j}, b_{j}\right] \rightarrow\left[a_{j}, b_{j}\right], h_{t}(\boldsymbol{O})=\boldsymbol{O}$, $J(t): I \rightarrow R^{1}$ defined by $J(t)=\partial H_{t}(\boldsymbol{O}) / \partial\left(x_{1}, \cdots, x_{n}\right)$ is a continuous nonvanishing function and $H_{t}$ is one-to-one on $V$, for all $t \in I$.

Since this result is stated without proof in [4, p. 215] one must assume that it is a well-known fact. However, for the convenience of the reader, a proof is given in the appendix below.

We can now prove Theorem 2. Choose $0 \in\left(a_{j}, b_{j}\right) \subset R^{1}$ so that $W=\prod_{j=1}^{n}\left[a_{j}, b_{j}\right]$ is contained in the open set $U$. Let $Y=f^{-1}(W) \cap W$, then there is an open subset of $R^{n}$ containing $O$ in $Y$. Extend $f \mid Y$, the restriction of $f$ to $Y$, to a map $k: W \rightarrow W$. By Lemma 9, there is a homotopy $h_{t}: W \rightarrow W$ such that $h_{0}=k, h_{1}=g_{1} \times \cdots \times g_{n}$ where $g_{j}:\left[a_{j}, b_{j}\right] \rightarrow\left[a_{j}, b_{j}\right], h_{t}(\boldsymbol{O})=\boldsymbol{O}$ and $H_{t}$ is one-to-one on a neighborhood $V$ of $O$, for all $t \in I$. Therefore $O$ is the only fixed point of each map $h_{t}$ on $V$ and, by the homotopy axiom, $i(W, k, \boldsymbol{O})=i\left(W, h_{1}, \boldsymbol{O}\right)$. 
Applying Lemma $8, i\left(W, h_{1}, \boldsymbol{O}\right)=\sigma\left(\partial H_{1}(\boldsymbol{O}) / \partial\left(x_{1}, \cdots, x_{n}\right)\right)$. Lemma 9 also states that $J(t)=\partial H_{t}(\boldsymbol{O}) / \partial\left(x_{1}, \cdots, x_{n}\right)$ is a continuous nonvanishing function of $t$ so $\sigma\left(\partial H_{1}(\boldsymbol{O}) / \partial\left(x_{1}, \cdots, x_{n}\right)\right)=\sigma\left(\partial H_{0}(\boldsymbol{O}) / \partial\left(x_{1}, \cdots, x_{n}\right)\right)$. But $h_{0}=k$ and $k$ agrees with $f$ near $\boldsymbol{O}$, therefore $\sigma\left(\partial H_{0}(O) / \partial\left(x_{1}, \cdots, x_{n}\right)\right)=$ $\sigma\left(\partial F(O) / \partial\left(x_{1}, \cdots, x_{n}\right)\right)$. On the other hand, if we extend $k$ to a map $k: X \rightarrow X$, then the commutativity axiom implies that $i(X, k, O)=$ $i(W, k, \boldsymbol{O})$. Applying the localization axiom, $i(X, f, \boldsymbol{O})=i(X, k, \boldsymbol{O})$. We have proved that $i(X, f, \boldsymbol{O})=\sigma\left(\partial F(\boldsymbol{O}) / \partial\left(x_{1}, \cdots, x_{n}\right)\right)$.

Appendix. Proof of Lemma 9. Write

$$
f\left(x_{1}, \cdots, x_{n}\right)=\left(f_{1}\left(x_{1}, \cdots, x_{n}\right), \cdots, f_{n}\left(x_{1}, \cdots, x_{n}\right)\right)
$$

where $f_{j}: W \rightarrow\left[a_{j}, b_{j}\right]$. Let $S(n)$ denote the symmetric group on $n$ symbols. For $\varphi \in S(n)$ define $h_{t}^{\varphi}: W \rightarrow W$ by

$$
h_{1-t}^{\varphi}\left(x_{1}, \cdots, x_{n}\right)=\left(f_{1}\left(t x^{\varphi(1)}\right), \cdots, f_{n}\left(t x^{\varphi(n)}\right)\right)
$$

where

$$
t x^{\varphi(j)}=\left(t x_{1}, \cdots, t x_{\varphi(j)-1}, x_{\varphi(j)}, t x_{\varphi(j)+1}, \cdots, t x_{n}\right) \in W .
$$

Let $F_{j k}=-\partial f_{j}(\boldsymbol{O}) / \partial x_{k}$ if $j \neq k$ and let $F_{j j}=1-\partial f_{j}(\boldsymbol{O}) / \partial x_{j}$ then

$$
\frac{\partial H_{1-t}^{\varphi}(\boldsymbol{O})}{\partial\left(x_{1}, \cdots, x_{n}\right)}=\pi(\varphi) F_{1 \varphi(1)} \cdots F_{n \varphi(n)}+\sum_{\psi \neq \varphi} \pi(\psi) t^{n} F_{1 \psi(1)} \cdots F_{n \psi(n)}
$$

where $\pi(\varphi)$ is 1 if $\varphi$ is an even permutation and -1 if $\varphi$ is odd. It is clear that $h_{0}^{\varphi}=f$ and that $h_{1}^{\varphi}=g_{1} \times \cdots \times g_{n}$ where

$$
g_{j}\left(x_{j}\right)=f_{k}\left(0, \cdots, 0, x_{j}, 0, \cdots, 0\right)
$$

and $\varphi(k)=j$. Also $J^{\varphi}(t)=\partial H_{t}^{\varphi}(\boldsymbol{O}) / \partial\left(x_{1}, \cdots, x_{n}\right)$ is certainly a continuous function of $t$.

However, there is a problem because there may exist $t_{\varphi} \in I$ such that $J^{\varphi}\left(t_{\varphi}\right)=0$. Observe first that the hypothesis $\partial F(\boldsymbol{O}) / \partial\left(x_{1}, \cdots, x_{n}\right) \neq 0$ implies that $t_{\varphi}<1$. Next note that we have in fact defined $n$ ! different homotopies $h_{t}^{\varphi}$, one for each element of $S(n)$. The question is whether there exist $n$ ! numbers $t_{\varphi}, 0 \leqq t_{\varphi}<1$, such that $J^{\varphi}\left(t_{\varphi}\right)=0$. We claim that there do not.

The system of equations $J^{\varphi}\left(t_{\varphi}\right)=0, \varphi \in S(n)$, can be written in the following form. Order the elements of $S(n)$ and let $\widetilde{\Im}=\left(F_{1}, \cdots, F_{n !}\right)$ where $F_{j}=F_{1 \varphi(1)} \cdots F_{n \varphi(n)}$ and $\phi$ is the $j$-th element of $S(n)$. Also write $t_{\varphi}=t_{j}$ in this case. The system can be written $T \mathfrak{\mho}=\boldsymbol{O}$ where

$$
T=T\left(t_{1}, \cdots, t_{n}\right)=\left[\begin{array}{cccc}
1 & t_{1} & \cdots & t_{1} \\
t_{2} & 1 & \cdots & t_{2} \\
\vdots & \vdots & & \vdots \\
t_{n !} & t_{n !} & \cdots & 1
\end{array}\right] \text {. }
$$


Since $\partial F(O) / \partial\left(x_{1}, \cdots, x_{n}\right) \neq 0$, it must be that $\mathfrak{F} \neq \boldsymbol{O}$ so there is a solution to $T \mathfrak{F}=\boldsymbol{O}$ if and only if $\operatorname{det}(T)=0$.

Observe that since all $t_{j}<1$ then

$$
\operatorname{det} T\left(1, t_{2}, \cdots, t_{n !}\right)=\prod_{j=2}^{n !}\left(1-t_{j}\right)>0 \text {. }
$$

If we write

$$
\operatorname{det} T\left(1, t_{2}, \cdots, t_{n !}\right)=\operatorname{det} T\left(t_{2}, \cdots, t_{n !}\right)-B_{n !}
$$

then

$$
B_{n !}=t_{2}\left(\operatorname{det} T\left(1, t_{3}, \cdots, t_{n !}\right)\right)+\cdots+t_{n !}\left(\operatorname{det} T\left(1, t_{2}, \cdots, t_{n !-1}\right)\right)
$$

so $B_{n !} \geqq 0$. Now

$$
\operatorname{det}(T)=\operatorname{det} T\left(t_{2}, \cdots, t_{n !}\right)-t_{1} B_{n !}
$$

so since $0 \leqq t_{j}<1$ for $j=2, \cdots, n$ ! then $\operatorname{det}(T)=0$ implies $B_{n !} \neq 0$ and

$$
t_{1}=\frac{\operatorname{det} T\left(t_{2}, \cdots, t_{n !}\right)}{B_{n !}}>1
$$

which establishes a contradiction and verifies the claim.

Thus there exists the required homotopy $h_{t}$ so that $\partial H_{t}(\boldsymbol{O}) / \partial\left(x_{1}, \cdots, x_{n}\right) \neq 0$ for all $t \in I$. It remains to find a single neighborhood $V$ of $O$ on which each $H_{t}$ is one-to-one. By hypothesis there exists $\delta>0$ such that if $p \in R^{n}$ and $|p| \leqq \delta$ then $f$ is of class $C^{1}$ at $p$. Let $B_{\delta}=\left\{p \in R^{n}|| p \mid \leqq \delta\right\}$ and define $D: B_{\delta} \times \cdots \times B_{\delta} \times I \rightarrow R^{1}$ by

$$
D\left(p_{1}, \cdots, p_{n}, t\right)=\operatorname{det}\left[\begin{array}{ccc}
\frac{\partial H_{t}\left(p_{1}\right)}{\partial x_{1}} & \cdots & \frac{\partial H_{t}\left(p_{1}\right)}{\partial x_{n}} \\
\vdots & \vdots \\
\frac{\partial H_{t}\left(p_{n}\right)}{\partial x_{1}} & \cdots & \frac{\partial H_{t}\left(p_{n}\right)}{\partial x_{n}}
\end{array}\right] .
$$

Note that $D(\boldsymbol{O} \times \cdots \times \boldsymbol{O} \times I) \subset R^{1}-0$. Let $C$ be the component of $D^{-1}\left(R^{1}-0\right)$ containing $\boldsymbol{O} \times \cdots \times \boldsymbol{O} \times I$, then there exists $\varepsilon>0$ such that $\left|p_{j}\right|<\varepsilon$ for $j=1, \cdots, n$ implies $\left(p_{1}, \cdots, p_{n}, t\right) \in C$ for all $t \in I$. Let $V=\left\{p \in R^{n}|| p \mid<\varepsilon\right\}$ then the Inverse Function Theorem tells us that each $H_{t}$ is one-to-one on $V$.

\section{REFERENCES}

1. F. Browder, On the fixed point index for continuous mappings of locally connected spaces, Summa Brasil. Math. 4 (1960), 253-293.

2. A. Dold, Fixed point index and fixed point theorem for Euclidean neighborhood retracts, Topology 4 (1965), 1-8. 
3. H. Hopf, Über die algebraische Anzahl von Fixpunkten, Math. Z. 29 (1929), 493-524. 4. J. Leray, Sur les équations et les transformations, J. Math. Pures et Appl. 24 (1945), 201-248.

5. B. O'Neill, Essential sets and fixed points, Amer. J. Math. 75 (1953), 497-509.

Received February 21, 1969. The preparation of this paper was supported in part by NSF Grant No. GP-6530.

University of CAlifornia, Los ANGeles 


\section{PACIFIC JOURNAL OF MATHEMATICS}

\section{EDITORS}

\author{
H. SAMELSON \\ Stanford University \\ Stanford, California 94305 \\ Richard Pierce \\ University of Washington \\ Seattle, Washington 98105
}

\author{
J. DugundJI \\ Department of Mathematics \\ University of Southern California \\ Los Angeles, California 90007 \\ RICHARD ARENS \\ University of California \\ Los Angeles, California 9.0024
}

\section{ASSOCIATE EDITORS}

\begin{tabular}{|c|c|}
\hline E. F. BECKENBACH & K. YoshidA \\
\hline \multicolumn{2}{|c|}{ SUPPORTING INSTITUTIONS } \\
\hline UNIVERSITY OF BRITISH COLUMBIA & STANFORD UNIVERSITY \\
\hline CALIFORNIA INSTITUTE OF TECHNOLOGY & UNIVERSITY OF TOKYO \\
\hline UNIVERSITY OF CALIFORNIA & UNIVERSITY OF UTAH \\
\hline MONTANA STATE UNIVERSITY & WASHINGTON STATE UNIVERSITY \\
\hline UNIVERSITY OF NEVADA & UNIVERSITY OF WASHINGTON \\
\hline NEW MEXICO STATE UNIVERSITY & * \\
\hline OREGON STATE UNIVERSITY & AMERICAN MATHEMATICAL SOCIETY \\
\hline UNIVERSITY OF OREGON & CHEVRON RESEARCH CORPORATION \\
\hline OSAKA UNIVERSITY & TRW SYSTEMS \\
\hline UNIVERSITY OF SOUTHERN CALIFORNIA & NAVAL WEAPONS CENTER \\
\hline
\end{tabular}

The Supporting Institutions listed above contribute to the cost of publication of this Journal, but they are not owners or publishers and have no responsibility for its content or policies.

Mathematical papers intended for publication in the Pacific Journal of Mathematics should be in typed form or offset-reproduced, (not dittoed), double spaced with large margins. Underline Greek letters in red, German in green, and script in blue. The first paragraph or two must be capable of being used separately as a synopsis of the entire paper. The editorial "we" must not be used in the synopsis, and items of the bibliography should not be cited there unless absolutely necessary, in which case they must be identified by author and Journal, rather than by item number. Manuscripts, in duplicate if possible, may be sent to any one of the four editors. Please classify according to the scheme of Math. Rev. Index to Vol. 39. All other communications to the editors should be addressed to the managing editor, Richard Arens, University of California, Los Angeles, California, 90024.

50 reprints are provided free for each article; additional copies may be obtained at cost in multiples of 50 .

The Pacific Journal of Mathematics is published monthly. Effective with Volume 16 the price per volume (3 numbers) is $\$ 8.00$; single issues, $\$ 3.00$. Special price for current issues to individual faculty members of supporting institutions and to individual members of the American Mathematical Society: $\$ 4.00$ per volume; single issues $\$ 1.50$. Back numbers are available.

Subscriptions, orders for back numbers, and changes of address should be sent to Pacific Journal of Mathematics, 103 Highland Boulevard, Berkeley, California, 94708.

PUBLISHED BY PACIFIC JOURNAL OF MATHEMATICS, A NON-PROFIT CORPORATION

Printed at Kokusai Bunken Insatsusha (Internatıonal Academic Printing Co., Ltd.), 7-17, Fujimi 2-chome, Chiyoda-ku, Tokyo, Japan. 


\section{Pacific Journal of Mathematics}

\section{Vol. 35, No. $3 \quad$ November, 1970}

John D. Arrison and Michael Rich, On nearly commutative degree one algebras . . . 533

Bruce Alan Barnes, Algebras with minimal left ideals which are Hilbert spaces . . . . 537

Robert F. Brown, An elementary proof of the uniqueness of the fixed point index . . . 549

Ronn L. Carpenter, Principal ideals in F-algebras .................... 559

Chen Chung Chang and Yiannis (John) Nicolas Moschovakis, The Suslin-Kleene

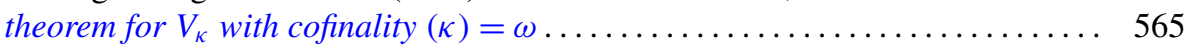

Theodore Seio Chihara, The derived set of the spectrum of a distribution

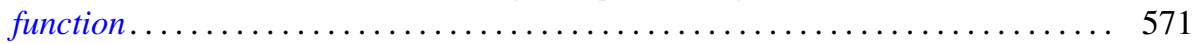

Tae Geun Cho, On the Choquet boundary for a nonclosed subspace of $C(S) \ldots \ldots \quad 575$

Richard Brian Darst, The Lebesgue decomposition, Radon-Nikodym derivative,

conditional expectation, and martingale convergence for lattices of sets .......

David E. Fields, Dimension theory in power series rings . . . . . . . . . . . .

Michael Lawrence Fredman, Congruence formulas obtained by counting

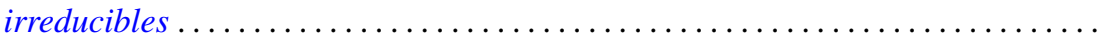

John Eric Gilbert, On the ideal structure of some algebras of analytic functions.....

G. Goss and Giovanni Viglino, Some topological properties weaker than

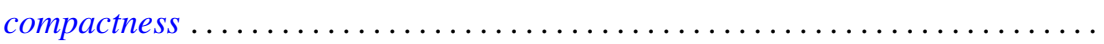

581

601

625

George Grätzer and J. Sichler, On the endomorphism semigroup (and category) of

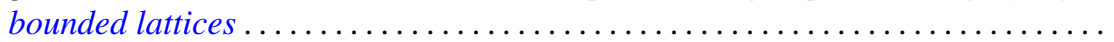

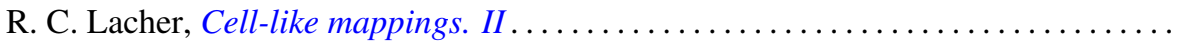

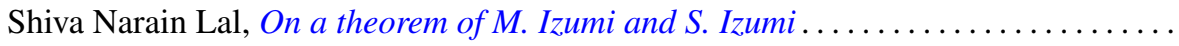

661

Howard Barrow Lambert, Differential mappings on a vector space ...............

Richard G. Levin and Takayuki Tamura, Notes on commutative power joined

semigroups.

Robert Edward Lewand and Kevin Mor McCrimmon, Macdonald's theorem for quadratic Jordan algebras.

J. A. Marti, On some types of completeness in topological vector spaces ....

Walter J. Meyer, Characterization of the Steiner point

717

Saad H. Mohamed, Rings whose homomorphic images are $q$-rings ...

727

Thomas V. O'Brien and William Lawrence Reddy, Each compact orientable surface

of positive genus admits an expansive homeomorphism ...

737

Robert James Plemmons and M. T. West, On the semigroup of binary relations...

743

Calvin R. Putnam, Unbounded inverses of hyponormal operator . .

755

William T. Reid, Some remarks on special disconjugacy criteria for differential

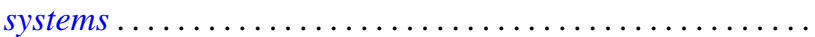

C. Ambrose Rogers, The convex generation of convex Borel sets in euclidean

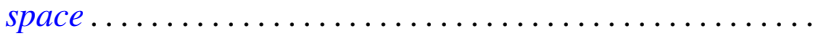

S. Saran, A general theorem for bilinear generating functions .

S. W. Smith, Cone relationships of biorthogonal systems ......

Wolmer Vasconcelos, On commutative endomorphism rings ....

795

Vernon Emil Zander, Products of finitely additive set functions from Orlicz

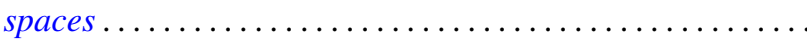

G. Sankaranarayanan and C. Suyambulingom, Correction to: "Some renewal

theorems concerning a sequence of correlated random variables" .

Joseph Zaks, Correction to: "Trivially extending decompositions of $E^{n}$ ”....... 805

Dong Hoon Lee, Correction to: "The adjoint group of Lie groups" ............ 805

James Edward Ward, Correction to: "Two-groups and Jordan algebras". 